\title{
Sonication of Arthroplasty Implants Improves Accuracy of Periprosthetic Joint Infection Cultures
}

\author{
Adam C. Rothenberg MD, Alan E. Wilson BS, John P. Hayes, \\ Michael J. O’Malley MD, Brian A. Klatt MD
}

Published online: 13 March 2017

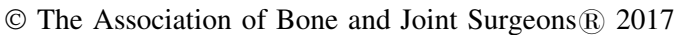

\begin{abstract}
Background There is evidence that sonication of explanted prosthetic hip and knee arthroplasty components with culture of the sonication fluid may enhance diagnostic sensitivity. Previous studies on the use of implant sonicate cultures have evaluated diagnostic thresholds but did not elaborate on the clinical importance of positive implant sonicate cultures in the setting of presumed aseptic revisions and did not utilize consensus statements on periprosthetic joint infection (PJI) diagnosis when defining their gold standard for infection.

Questions/purposes (1) How do implant sonicate cultures compare with preoperative synovial fluid cultures and intraoperative tissue cultures in the diagnosis of PJI in both THA and TKA when compared against Musculoskeletal Infection
\end{abstract}

Each author certifies that neither he, nor any member of his immediate family, have funding or commercial associations (consultancies, stock ownership, equity interest, patent/licensing arrangements, etc) that might pose a conflict of interest in connection with the submitted article.

All ICMJE Conflict of Interest Forms for authors and Clinical Orthopaedics and Related Research ${ }^{\mathbb{R}}$ editors and board members are on file with the publication and can be viewed on request.

Clinical Orthopaedics and Related Research ${ }^{\circledR}$ neither advocates nor endorses the use of any treatment, drug, or device. Readers are encouraged to always seek additional information, including FDAapproval status, of any drug or device prior to clinical use. Each author certifies that his institution approved the human protocol for this investigation, that all investigations were conducted in conformity with ethical principles of research, and that informed consent for participation in the study was obtained.

A. C. Rothenberg ( $₫)$, A. E. Wilson, J. P. Hayes,

M. J. O’Malley, B. A. Klatt

Department of Orthopaedic Surgery, University of Pittsburgh,

3471 5th Avenue, Suite 1010, Pittsburgh, PA 15213, USA

e-mail: rothenbergac@upmc.edu
Society (MSIS) criteria for PJI? (2) Utilizing implant sonicate cultures, what is the relative prevalence of bacterial species identified in PJIs? (3) What is the incidence of positive implant sonicate cultures in the setting of presumed aseptic revision hip and knee arthroplasty procedures, and what treatments did they receive?

Methods Between 2012 and 2016 we performed implant sonicate fluid cultures on surgically removed implants from 565 revision THAs and TKAs. Exclusion criteria including insufficient data to determine Musculoskeletal Infection Society (MSIS) classification, fungal-only cultures, and absence of reported colony-forming units decreased the number of procedures to 503. Procedures represented each instance of revision surgery (sometimes multiple in the same patient). Of those, a definitive diagnosis of infection was made using the MSIS criteria in 178 of 503 (35\%), whereas the others (325 of 503 [65\%]) were diagnosed as without infection. A total of 53 of 325 (16\%) were considered without infection based on MSIS criteria but had a positive implant sonicate culture. Twenty-five of 53 (47\%) of these patients were followed for at least 2 years. The diagnosis of PJI was determined using the MSIS criteria.

Results Sensitivity of implant sonicate culture was greater than synovial fluid culture and tissue culture $(97 \%$ [89\%-99\%] versus 57\% [44\%-69\%], p < 0.001; 97\% [89\%-99\%] versus $70 \%$ [58\%-80\%], $\mathrm{p}<0.001$, respectively). The specificity of implant sonicate culture was not different from synovial fluid culture or tissue culture with the numbers available (90\% [72\%-97\%] versus $100 \%$ [86\%-100\%], $\mathrm{p}=0.833 ; 90 \%[72 \%-97 \%]$ versus $97 \%$ [81\%-100\%], $\mathrm{p}=0.317$, respectively). Coagulase-negative Staphylococcus was the most prevalent organism for both procedure types. In PJIs, the five most frequent bacteria identified by synovial fluid, tissue, and/or implant sonicate cultures were coagulase-negative Staphylococcus (26\% [89 
of 267]), methicillin-susceptible Staphylococcus aureus (19\% [65 of 267]), methicillin-resistant S. aureus (12\% [43 of 267]), $\alpha$-hemolytic Streptococci (5\% [19 of 267]), and Enterococcus faecalis (5\% [19 of 267]). Fifty-three of 325 (16\%) presumed aseptic revisions had a positive sonication culture. Thirty-four percent (18 of 53) of culture-positive aseptic revision patients received antibiotic treatment for infection and $8 \%$ (4 of 53) underwent a secondary revision. Conclusions The routine use of implant sonicate cultures in arthroplasty revisions improves the diagnostic sensitivity for detecting the presence of bacteria in both clinical and occult infections. Future studies will need to refine colonyforming unit thresholds for determining clinical infection and indications for treatment.

Level of Evidence Level III, diagnostic study.

\section{Introduction}

A major challenge in management of periprosthetic joint infections (PJIs) is accurate identification of causative organisms and their respective sensitivities. Rates of successful eradication of PJI are improved when the causative organism is known [18]. Definitions of PJI rely on serum inflammatory markers, arthrocentesis-based studies, and intraoperative tissue cultures. Preoperative laboratory studies such as $\alpha$-defensin, erythrocyte sedimentation rate, and $\mathrm{C}$-reactive protein can indicate the presence of infection but are not able to guide antimicrobial therapy. Synovial fluid cultures and intraoperative tissue cultures are the current standard for detecting bacterial speciation but have an unacceptably high false-negative rate (17\%$53 \%)$ [1, 2, 15]. Polymerase chain reaction-based methodologies have shown potential as a means of rapid molecular diagnosis but at this time lack the ability to reliably provide antibiotic sensitivities $[3,4]$. Culturing the fluid from sonicated prosthetic implants has been previously investigated as an adjunctive method for diagnosing PJI, but its use has not become widespread as a result of questions about its relative accuracy compared with standard culture types.
The role of implant sonicate culture as an adjunct diagnostic tool continues to evolve. An inherent advantage of sonication is the ability to disrupt bacteria present within biofilms, increasing the number of culturable bacterial cells. Sonication may help to identify a broader spectrum of infectious organisms that exist primarily within protected biofilm structures in procedures otherwise characterized as aseptic failures. Occult infections can be responsible for implant loosening, chronic pain, and instability and may be underdiagnosed by traditional culture methods $[6,7,13]$. Multiple studies have evaluated the performance of sonication fluid culture with reported sensitivities ranging from $73 \%$ to $88 \%$ and specificities from $87 \%$ to $99 \%$ [4, 5, 10-12, 14, 16]. However, prior investigations did not utilize newer consensus statements on PJI diagnosis when defining their gold standard for infection, raising questions about the accuracy of their PJI diagnoses and the analyses that followed from those diagnoses (Table 1).

We therefore asked: (1) How do implant sonicate cultures compare with preoperative synovial fluid cultures and intraoperative tissue cultures in the diagnosis of PJI in both THA and TKA when compared against Musculoskeletal Infection Society (MSIS) criteria for PJI (Table 1)? (2) Utilizing implant sonicate cultures, what is the relative prevalence of bacterial species identified in PJIs? (3) What is the incidence of positive implant sonicate cultures in the setting of presumed aseptic revision hip and knee arthroplasty procedures, and what treatments did they receive?

\section{Patients and Methods}

A retrospective study was performed utilizing an institutional review board-approved Joints Outcomes Registry Database of 565 revision total hip or knee arthroplasty procedures in 468 patients indicated for infection or aseptic revision at the University of Pittsburgh Medical Center (urban, academic) hospitals in Pittsburgh, PA, USA,

Table 1. Musculoskeletal Infection Society 2014 Consensus definition of periprosthetic joint infection (PJI) [8]

$\mathrm{PJI}$ is present when one of the major criteria exists or three of five minor criteria exist

Major criteria Two positive periprosthetic cultures with phenotypically identical organisms, OR

A sinus tract communicating with the joint, OR

Minor criteria 1. Serum erythrocyte sedimentation rate $\geq 30 \mathrm{~mm} / \mathrm{hr}$ AND C-reactive protein $\geq 1 \mathrm{mg} / \mathrm{dL}$

2. Synovial fluid white blood cell count $\geq 3000$ OR ++ change on leukocyte esterase test strip

3. Synovial fluid polymorphonuclear neutrophil percentage $\geq 80 \%$

4. Positive histologic analysis of periprosthetic tissue ( $>5$ neutrophils per high-power field in 5 high-power fields $(\times 400)$

5. A single positive culture

$\mathrm{PJI}$ is defined by the presence of at least one major criterion and/or at least three minor criteria. 
between October 2012 and May 2016. Procedures represented each instance of revision surgery (sometimes multiple in the same patient over the 4-year study period). Exclusion criteria including insufficient data to determine MSIS classification, fungal-only cultures, and absence of reported colony-forming units narrowed the procedure number to 503 (Fig. 1). Of those, a definitive diagnosis of infection was made using the MSIS consensus criteria in 178 of 503 (35\%), whereas the others (325 of 503 [65\%]) were diagnosed as without infection (Table 1) [8, 9]. A total of 53 of $325(16 \%)$ were considered without infection based on MSIS criteria but had positive implant sonicate culture. This subgroup of patients was followed longitudinally and 25 of $53(47 \%)$ were followed for at least 2 years.

Clinical screening including serum and synovial fluid assays to either include or exclude PJI was initiated in the preoperative phase. Inclusion criteria included all patients who had implant sonicate cultures performed on explanted arthroplasty components over a 4-year period. Implant sonicate cultures were performed in all revision arthroplasty procedures (both infected and aseptic), but a small number (5 of 565 [3\%]) of sonication cultures were invalidated by improper handling methods and were thus excluded from analysis. Aseptic revisions were defined as procedures requiring revision resulting from aseptic failure (such as mechanical symptoms, evidence of implant loosening, and/or implant malpositioning) in the absence of clinical suspicion for infection and not otherwise satisfying consensus criteria for PJI. Sonication of antibiotic-impregnated spacers from two-stage revision procedures was also excluded from this analysis.

\section{Tissue/Synovial Culture Method}

Tissues were homogenized using a disposable tissue homogenizer in Trypticase soy broth (BD Microbiology Systems, Sparks, MD, USA) in an approximately $10 \%$
Fig. 1 The flow diagram outlines the figures involved in applying patient inclusion and exclusion criteria and defining procedure subtypes.
565 Cases Treated for Periprosthetic Joint Infection or Presumed Aseptic Revision (October 2012 to May 2016)

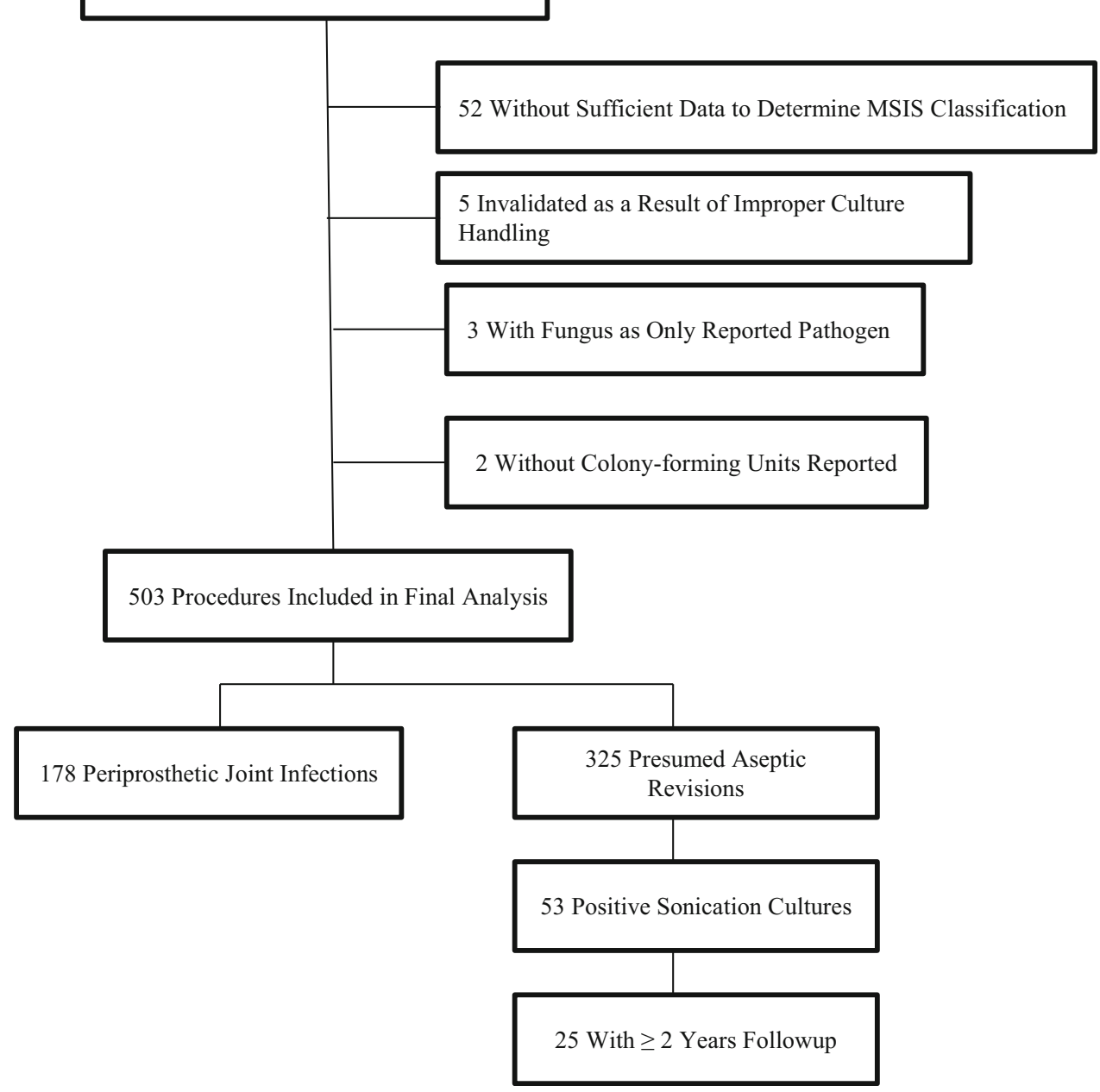


(weight/volume) suspension. One-tenth milliliter of this suspension was inoculated onto blood agar, colistin nalidixic acid agar, MacConkey agar, and chocolate agar (BD Microbiology Systems). The aerobic cultures were incubated for 48 hours at $37^{\circ} \mathrm{C}$. If these aerobic cultures were negative at 48 hours, the chocolate plate was incubated for a third day before calling the culture negative. Anaerobic cultures of homogenized tissue were inoculated onto prereduced anaerobically sterilized Brucella, phenylethyl alcohol, and kanamycin vancomycin laked blood agars (Hardy Diagnostics, Santa Maria, CA, USA). The inoculated plates were incubated in an anaerobic jar at $35^{\circ} \mathrm{C}$ for 48 hours. When isolation of Propionibacterium acnes was requested, the anaerobic plates were incubated for 14 days. During the first 2 years, organisms were identified using standard phenotypic methods. More recently, aerobic and anaerobic organisms were identified using matrix-assisted laser desorption ionization time-of-flight mass spectrometry. Synovial fluids were cultured similarly, aerobically, and anaerobically, except that $0.1 \mathrm{~mL}$ of fluid was inoculated onto each plate.

\section{Sonication Procedure}

The sonication protocol was adapted from Cazanave et al. [4]. Removed implant material was placed into Nalgene jars containing $400 \mathrm{~mL}$ lactated Ringer's solution. The jar was transferred to a degassed sonication water bath $(40 \pm 2$ $\mathrm{kHz}, 0.22 \mathrm{~W} / \mathrm{cm}^{2}$ ) and sonicated for 5 minutes. When sonication was completed, the jar was allowed to sit for 1 minute to allow any aerosol to settle. The container was then vortexed for 30 seconds and transferred to a biologic safety cabinet. Inside the cabinet, the container and lid were wiped down using a gauze square saturated with alcohol. Using an irrigation syringe, the entire volume of sonicated fluid was decanted into $50-\mathrm{mL}$ conical centrifuge tubes. The tubes were centrifuged at $4^{\circ} \mathrm{C}$ at $3150 \times \mathrm{g}$ for 5 minutes. All tubes were removed from the centrifuge and the supernatant was decanted and discarded except for the last $1 \mathrm{~mL}$ of fluid in each tube. The tubes were vortexed to resuspend each sediment in $1 \mathrm{~mL}$ of supernatant and combined in a single centrifuge tube, which was again centrifuged for 5 minutes. The supernatant was decanted except for the last $4 \mathrm{~mL}$ and the pellet was resuspended in this volume. One-tenth milliliter volumes of the suspension were inoculated onto a blood agar plate and a chocolate agar plate, which were incubated in $5 \% \mathrm{CO}_{2}$ for 4 days. Prereduced anaerobically sterilized Brucella agar and phenylethyl alcohol agar were inoculated with identical volumes of specimen, which were placed in anaerobic boxes and incubated at $35^{\circ} \mathrm{C}$ for 14 days. During the first 2 years, organisms were identified by standard phenotypic methods. More recently, most organisms were identified using matrix-assisted laser desorption ionization time-offlight mass spectrometry.

Demographic variables recorded included age, sex, joint, and laterality of the joint. The preoperative diagnosis, procedure, and operating surgeon were also recorded. Preoperative variables recorded included erythrocyte sedimentation rate, C-reactive protein, synovial fluid leukocyte count and differential, and synovial fluid culture results. The presence of a draining sinus tract was also documented. Intraoperative and postoperative variables included tissue culture results, histologic analysis of tissue (neutrophils/high-power field), type of sonicated material sonication culture result and colony-forming units, and sonication culture pathogen. Additional variables recorded for presumed aseptic revision procedures with positive implant sonicate cultures were described previously.

\section{Treatment of Patients With Contaminants}

Patients who underwent presumed aseptic revision with positive implant sonicate cultures were followed longitudinally. Initiation of antibiotic therapy was recorded as documented in the postoperative or infectious disease consult notes. Patient outcome data were gathered from postoperative clinic notes from 2-week, 6-week, 3-month, 6-month, 1-year, and 2-year appointments (whenever possible). Additional screening included chart review of all available records for all patients at the time of most recent clinical followup within the University of Pittsburgh Medical Center Hospital Health System. Infectious disease clinic and primary care provider documentation was reviewed as indicated. All patient charts were reviewed for additional surgical procedures performed on the index joint during the followup period. The date, indication, and type of procedure were recorded for these instances. Length of followup was calculated as the difference between date of surgery and the most recent date of contact with a healthcare provider. Antibiotic administration was determined on a case-by-case basis in conjunction with Infectious Diseases regarding clinical suspicion of infection in the case of aseptic revisions with positive implant sonicate cultures. Clinical judgment incorporating impression of the likelihood of infection by the operating surgeon as well as clinical history including but not limited to antecedent infection history was incorporated into the decision to treat a procedure with a positive implant sonicate culture as a PJI. Indications for surgical intervention 
for PJI included patients meeting MSIS consensus criteria for infection.

\section{Statistical Analysis}

Sensitivity, specificity, positive predictive value, and negative predictive value of microbiologic cutoffs were calculated with two-by-two contingency tables. The optimal colony-forming unit (CFU) threshold (per agar plate) was determined using Youden's J statistic, calculated as J = sensitivity + specificity -1 for each possible CFU/plate threshold value. Greater than or equal to $5 \mathrm{CFU} /$ plate was selected as the optimal cutpoint based on Youden's J statistic. All implant sonicate culture sensitivities and specificities reported here were calculated using this threshold. Receiver operating characteristic analysis was performed using SPSS Statistics software (IBM Corp, Armonk, NY, USA). As a retrospective study, not all culture types were collected for each patient, particularly in the presumed aseptic revision group that had a low clinical suspicion for infection. In 97 of 503 patients (19\%), all three culture types were collected and subgroup analysis was performed for direct comparison. McNemar's test was applied to paired data to evaluate statistically significant differences in diagnostic accuracy for implant sonicate culture versus tissue culture and implant sonicate culture versus synovial fluid culture.

A total of 503 procedures met inclusion criteria for this study (Table 2). Of these, 178 were PJIs, whereas 325 were presumed aseptic revisions. The PJIs included a greater percentage of males and were more likely to involve the knee than the hip. Erythrocyte sedimentation rate and Creactive protein were elevated in $88 \%$ and $85 \%$ of PJIs, respectively, versus $22 \%$ and $22 \%$ of presumed aseptic revisions.

\section{Results}

The area under the receiver operating characteristic curve for sonication fluid culture was 0.944 (95\% confidence interval, 0.920-0.969; Fig. 2). The sensitivities of implant sonicate culture, tissue culture, and synovial fluid culture

Table 2. Patient demographics for all patients enrolled in the study

\begin{tabular}{|c|c|c|}
\hline Characteristic & $\begin{array}{l}\text { Prosthetic joint infection } \\
(\mathrm{n}=178)\end{array}$ & $\begin{array}{l}\text { Aseptic revision } \\
(\mathrm{n}=325)\end{array}$ \\
\hline \multicolumn{3}{|l|}{ Age (years) } \\
\hline Median & 64.0 & 65.6 \\
\hline Range & $19-89$ & $22-91$ \\
\hline \multicolumn{3}{|l|}{ Sex, number $(\%)$} \\
\hline Male & $98(55 \%)$ & $147(45 \%)$ \\
\hline Female & $80(45 \%)$ & $178(55 \%)$ \\
\hline \multicolumn{3}{|l|}{ Site of arthroplasty, number $(\%)$} \\
\hline Hip & $62(35 \%)$ & $178(55 \%)$ \\
\hline Knee & $116(65 \%)$ & $147(45 \%)$ \\
\hline \multicolumn{3}{|l|}{ Type of revision, number $(\%)$} \\
\hline Débridement with prosthesis retention & $30(17 \%)$ & $0(0 \%)$ \\
\hline One-stage & $20(11 \%)$ & $323(99 \%)$ \\
\hline Two-stage & $128(72 \%)$ & $2(1 \%)$ \\
\hline \multicolumn{3}{|l|}{ Sonicated material, number $(\%)$} \\
\hline Implant & $146(82 \%)$ & $294(91 \%)$ \\
\hline Modular component & $32(18 \%)$ & $31(10 \%)$ \\
\hline Presence of sinus tract, number $(\%)$ & $46(26 \%)$ & $0(0 \%)$ \\
\hline \multicolumn{3}{|c|}{ Preoperative laboratory values, number/total number (\%) } \\
\hline Erythrocyte sedimentation rate $>30 \mathrm{~mm} / \mathrm{hr}$ & $132 / 150(88 \%)$ & $42 / 191(22 \%)$ \\
\hline Serum C-reactive protein $>1.0 \mathrm{mg} / \mathrm{dL}$ & $126 / 149(85 \%)$ & $42 / 189(22 \%)$ \\
\hline Synovial fluid leukocyte count $>3000$ cells & $111 / 119(93 \%)$ & $12 / 93(13 \%)$ \\
\hline Synovial fluid differential $>80 \%$ neutrophils & $86 / 95(91 \%)$ & $7 / 49(14 \%)$ \\
\hline \multicolumn{3}{|c|}{ Histologic analysis of tissue, number/total number $(\%)$} \\
\hline$>5$ neutrophils per HPF in 5 HPF $(\times 400)$ & $35 / 51(71 \%)$ & $2 / 69(3 \%)$ \\
\hline
\end{tabular}

HPF $=$ high-power field. 
Fig. 2 The graph and associated table demonstrate the receiver operating characteristic (ROC) curve for implant sonicate cultures. The test result variable(s): sonication colonies have at least one tie between the positive actual state group and the negative actual state group; statistics may be biased; *under the nonparametric assumption; ${ }^{\dagger}$ null hypothesis: true area $=0.5$.
Area under the curve

Test result variable(s): sonication colonies

\begin{tabular}{|c|c|c|c|c|}
\hline Area & Standard error $^{*}$ & Asymptotic significance $^{\dagger}$ & \multicolumn{2}{|c|}{ Asymptotic 95\% confidence interval } \\
\cline { 3 - 5 } & & & Lower bound & Upper bound \\
\hline 0.944 & 0.012 & 0.000 & 0.920 & 0.969 \\
\hline
\end{tabular}

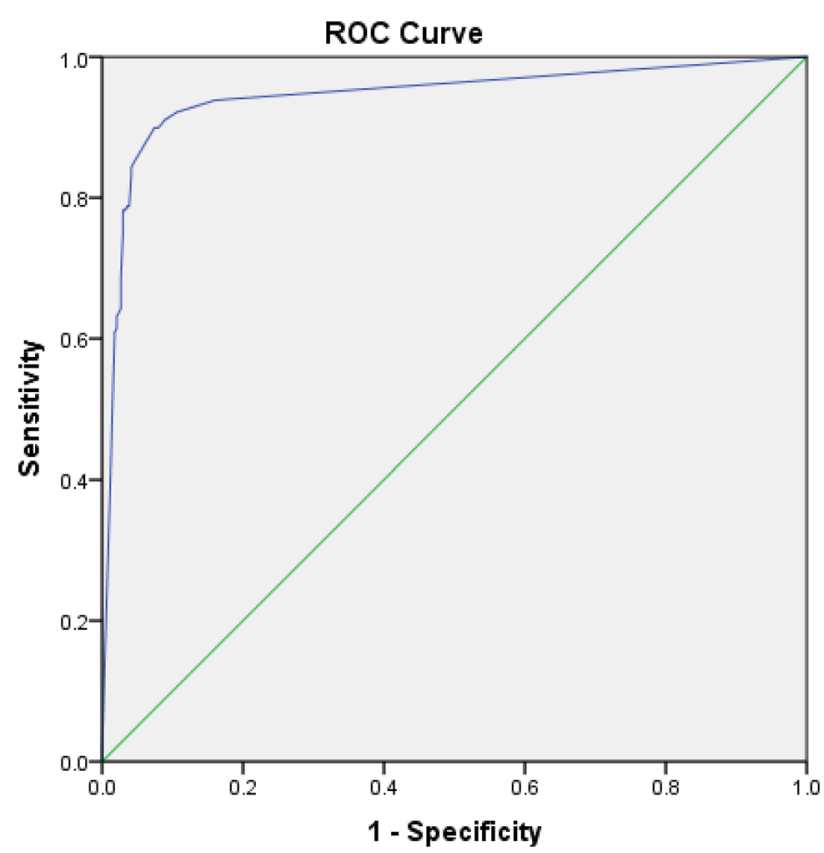

Diagonal segments are produced by ties.

Table 3. An outline of periprosthetic joint infection diagnosis by culture type*

\begin{tabular}{|c|c|c|c|c|c|c|}
\hline Test & Prosthetic joint infection & Aseptic revision & Sensitivity & Specificity & PPV & NPV \\
\hline Synovial fluid culture & $72 / 131$ & $0 / 94$ & $55 \%$ & $100 \%$ & $100 \%$ & $61 \%$ \\
\hline Tissue culture & $74 / 104$ & $1 / 56$ & $71 \%$ & $98 \%$ & $99 \%$ & $65 \%$ \\
\hline \multicolumn{7}{|l|}{ Implant sonicate culture } \\
\hline$\geq 1 \mathrm{CFU}^{\dagger}$ & $167 / 178$ & $53 / 325$ & $94 \%$ & $84 \%$ & $76 \%$ & $96 \%$ \\
\hline$\geq 2$ CFUs & $164 / 178$ & $35 / 325$ & $92 \%$ & $89 \%$ & $82 \%$ & $95 \%$ \\
\hline$\geq 3$ CFUs & $162 / 178$ & $29 / 325$ & $91 \%$ & $91 \%$ & $85 \%$ & $95 \%$ \\
\hline$\geq 4$ CFUs & $160 / 178$ & $26 / 325$ & $90 \%$ & $92 \%$ & $86 \%$ & $94 \%$ \\
\hline$\geq 5$ CFUs & $160 / 178$ & $24 / 325$ & $90 \%$ & $93 \%$ & $87 \%$ & $94 \%$ \\
\hline$\geq 10$ CFUs & $148 / 178$ & $14 / 325$ & $83 \%$ & $96 \%$ & $91 \%$ & $91 \%$ \\
\hline$\geq 25$ CFUs & $122 / 178$ & $9 / 325$ & $69 \%$ & $97 \%$ & $93 \%$ & $85 \%$ \\
\hline
\end{tabular}

* Included are statistics including absolute and percentages for synovial fluid cultures, tissue cultures, and implant sonication cultures, including stratification of results by colony-forming unit (CFU) per agar plate thresholds for the latter; the optimal CFU cutoff as determined by receiver operating characteristic analysis was determined to be $\geq 5 \mathrm{CFUs}$ (per agar plate); ${ }^{\dagger} \mathrm{CFU} /$ agar plate represents a dilution of $400 \mathrm{~mL}$ LR solution to a final plating of $0.1-\mathrm{mL}$ aliquots after centrifugation; PPV = positive predictive value; NPV = negative predictive value.

were $90 \%(84 \%-94 \%), 71 \%(61 \%-79 \%)$, and $55 \%(46 \%-$ $64 \%$ ), respectively (Table 3 ). The specificities of implant sonicate culture, tissue culture, and synovial fluid culture were $93 \%(88 \%-95 \%), 98 \%(89 \%-100 \%)$, and $100 \%$ (95\%-100\%), respectively. A subgroup analysis of 97 procedures in which patients had all three culture types collected was performed for direct comparison (Table 4). The sensitivity of implant sonicate culture was greater than synovial fluid culture and tissue culture (97\% [89\%-99\%] versus 57\% [44\%-69\%], p < 0.001; 97\% [89\%-99\%] versus $70 \%$ [58\%-80\%], $\mathrm{p}<0.001$, respectively). The specificity of implant sonicate culture was not different 
from the synovial fluid culture or tissue culture with the numbers available (90\% [72\%-97\%] versus $100 \%$ [86\%$100 \%], \mathrm{p}=0.833 ; 90 \%[72 \%-97 \%]$ versus $97 \%[81 \%-$ $100 \%$ ], $\mathrm{p}=0.317$, respectively). Implant sonicate culture of prosthetic hip components had a sensitivity of $89 \%$ (55 of $62[78 \%-95 \%])$ and specificity of $93 \%$ (166 of 178 [88\%$96 \%]$ ), whereas prosthetic knee components had a sensitivity of $91 \%$ (105 of 116 [83\%-95\%]) and specificity of $92 \%$ (135 of 147 [87\%-96\%]).

The pathogens most commonly identified by implant sonicate culture were different for patients with PJI versus those with presumed aseptic revision (Table 5). Coagulase-negative Staphylococcus was the most prevalent organism for both procedure types. In PJIs, the five most frequent bacteria identified by synovial fluid, tissue, and/ or implant sonicate cultures were coagulase-negative Staphylococcus (26\% [89 of 267]), methicillin-susceptible Staphylococcus aureus (MSSA; 19\% [65 of 267]), methicillin-resistant $S$ aureus (MRSA; 12\% [43 of 267]), $\alpha$-hemolytic Streptococci (5\% [19 of 267]), and Enterococcus faecalis (5\% [19 of 267]). Within aseptic revisions the five most frequent bacteria identified were coagulasenegative Staphylococcus (41\% [26 of 64]), Propionibacterium acnes (14\% [nine of 64]), diphtheroids (12\% [seven of 64]), Micrococcus (6\% [four of 64]), and Peptostreptococcus (5\% [three of 64]).

Fifty-three of 325 (16\%) patients undergoing presumed aseptic revision had a positive sonication culture. Twentyfour of 325 cultures $(8 \%)$ had $\geq 5 \mathrm{CFUs} /$ plate. Eighteen of 53 patients $(34 \%)$ with positive sonication cultures were

Table 4. Subgroup analysis of patients who had all three culture types available for direct comparison

\begin{tabular}{lll}
\hline Culture type & Sensitivity & Specificity \\
\hline Synovial fluid culture & $57 \%(38 / 67)$ & $100 \%(30 / 30)$ \\
Tissue culture & $70 \%(47 / 67)$ & $97 \%(29 / 30)$ \\
Implant sonicate culture & $97 \%(65 / 67)$ & $90 \%(27 / 30)$ \\
\hline
\end{tabular}

treated with antibiotics. Although no patients in this cohort were lost to followup, mean length of followup was 22 months (SD 11 months) at the time of reporting. At this time, 25 patients have been followed for at least 2 years (Table 6). Four of these patients ultimately underwent surgical intervention for treatment of infection during the followup period including three with greater than 5 $\mathrm{CFU} /$ plate. Of the remaining aseptic revision patients with positive implant sonicate cultures who did not require reoperation, three of seven $(42 \%)$ received antibiotic treatment.

\section{Discussion}

Implant sonicate culture enhances the diagnostic assessment for PJI by identifying pathogens that are inaccessible to traditional intraoperative tissue and synovial fluid cultures. By amplifying the sampling of microbiologic cellular material, sonication improves the sensitivity of cultures similarly as alternative methods such as polymerase chain reaction or other molecular-based diagnostic methods while also providing antibiotic sensitivity testing [3, 4]. Previous reports on the use of implant sonication culture in revision arthroplasty have attempted to determine the sensitivity of this diagnostic method but have not uniformly applied the MSIS consensus criteria as a determinant of infection [4, 5, 10-12, 14, 16]. The definition of the microbiologic threshold for a clinically important result and specific indications for its use are emerging but still incomplete. The utility of applying this method to patients with presumed aseptic revisions where there is a low pretest suspicion for the presence of a microorganism is particularly ill-defined.

Limitations of this study include the inherent flaws in a retrospective, database study including but not limited to incomplete patient information. First, not all elements of MSIS PJI criteria were available for all patients and their preoperative measurement varied in the timeframe when

Table 5. Relative incidence of bacterial species stratified by case type

\begin{tabular}{llll}
\hline Aseptic revision & & & Periprosthetic joint infection \\
Pathogen & Frequency $(\%)$ & Pathogen & Frequency $(\%)$ \\
\hline CoNS & 41 & CoNS & 26 \\
Propionibacterium acnes & 14 & MSSA & 19 \\
Diphtheroids & 10 & MRSA & 12 \\
Micrococcus & 6 & $\alpha$-hemolytic Streptococci & 5 \\
Peptostreptococcus & 5 & Enterococcus faecalis & 5 \\
$\alpha$-hemolytic Streptococci & 5 & Group B Streptococci & 5 \\
\hline
\end{tabular}

CoNS = coagulase-negative Staphylococcus; MSSA = methicillin-susceptible Staphylococcus aureus; MRSA = methicillin-resistant $S$ aureus. 
Table 6. Test results and clinical outcomes of aseptic revision cases that had positive sonication fluid cultures

\begin{tabular}{|c|c|c|c|c|c|c|c|c|}
\hline $\begin{array}{l}\text { Case } \\
\text { number }\end{array}$ & Diagnosis & $\begin{array}{l}\text { ESR } \\
(\mathrm{mm} / \mathrm{hr})\end{array}$ & $\begin{array}{l}\text { CRP } \\
(\mathrm{mg} / \mathrm{dL})\end{array}$ & CFUs & Organism & Antibiotics & $\begin{array}{l}\text { Length of } \\
\text { followup (years) }\end{array}$ & $\begin{array}{l}\text { Additional surgery } \\
\text { for infection }\end{array}$ \\
\hline 1 & Revision & 14 & 0.13 & 1 & $\begin{array}{l}\text { Propionibacterium } \\
\text { acnes }\end{array}$ & - & 2.1 & - \\
\hline 2 & Revision & 17 & 0.15 & 1 & $\begin{array}{l}\text { Propionibacterium } \\
\text { acnes }\end{array}$ & - & 2.1 & - \\
\hline 3 & Revision & 2 & 0.1 & 1 & CoNS & - & 2.3 & - \\
\hline 4 & Revision & - & 0.29 & 1 & CoNS & - & 2.4 & - \\
\hline 5 & Revision & - & - & 1 & CoNS & Daptomycin & 3.0 & - \\
\hline 6 & Revision & - & - & 1 & CoNS & - & 2.7 & - \\
\hline 7 & Revision & - & - & 1 & Diphtheroids & - & 2.2 & - \\
\hline 8 & Revision & 23 & 0.18 & 1 & Micrococcus & - & 2.1 & - \\
\hline 9 & Revision & - & - & 1 & Micrococcus & - & 2.5 & - \\
\hline 10 & Revision & - & - & 2 & CoNS & Vancomycin, rifampin & 2.0 & - \\
\hline 11 & Revision & 6 & 0.46 & 2 & CoNS & Vancomycin & 2.2 & - \\
\hline 12 & Revision & - & - & 2 & CoNS & - & 2.3 & - \\
\hline 13 & Revision & 10 & 0.3 & 3 & Diphtheroids & - & 2.1 & - \\
\hline 14 & Revision & - & - & 3 & MRSA & Vancomycin, rifampin & 2.7 & - \\
\hline 15 & Revision & 10 & 0.19 & 4 & CoNS & - & 2.9 & $\mathrm{I} \& \mathrm{D}$ \\
\hline 16 & Revision & 2 & 0.1 & 5 & CoNS & - & 2.0 & $\mathrm{I} \& \mathrm{D}$ \\
\hline 17 & Revision & - & - & 5 & $\begin{array}{l}\text { Propionibacterium } \\
\quad \text { acnes }\end{array}$ & - & 2.5 & - \\
\hline 18 & Revision & 13 & 0.3 & 5 & Actinomyces meyeri & - & 2.6 & - \\
\hline 19 & Revision & - & - & 5 & CoNS & Vancomycin & 3.0 & - \\
\hline 20 & Revision & - & - & 5 & CoNS & - & 2.6 & - \\
\hline 21 & Revision & 8 & 0.07 & 11 & $\begin{array}{l}\text { Propionibacterium } \\
\text { acnes }\end{array}$ & Augmentin & 2.1 & $I \& D$ \\
\hline 22 & Revision & - & - & $\begin{array}{l}50, \\
30\end{array}$ & $\begin{array}{l}\text { Proteus mirabilis, } \\
\text { MRSA }\end{array}$ & $\begin{array}{l}\text { Vancomycin, } \\
\text { ceftriaxone }\end{array}$ & 2.1 & $I \& D$ \\
\hline 23 & Revision & 12 & 0.16 & 75 & CoNS & - & 2.6 & - \\
\hline 24 & Revision & 44 & 0.99 & $\begin{array}{l}100, \\
3\end{array}$ & $\begin{array}{l}\text { CoNS } \\
\text { Diphtheroids }\end{array}$ & $\begin{array}{l}\text { Ceftriaxone, } \\
\text { cephalexin }\end{array}$ & 2.2 & - \\
\hline 25 & Revision & 8 & 0.20 & $\begin{array}{l}100 \\
100 \\
100\end{array}$ & $\begin{array}{l}\text { Diphtheroids } \\
\text { Micrococcus } \\
\text { Viridans Streptococci }\end{array}$ & - & 3.1 & - \\
\hline
\end{tabular}

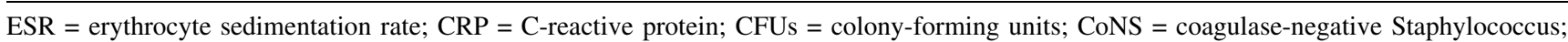
MRSA = methicillin-resistant Staphylococcus aureus; I\&D = irrigation and débridement.

they were performed. Erythrocyte sedimentation rate and C-reactive protein values were available for $68 \%$ (341 of 503 ) and $67 \%$ (338 of 503) of patient procedures, respectively. Synovial fluid culture was collected from $45 \%$ of patient procedures (225 of 503) with synovial fluid cell count and differential reported for $42 \%$ (212 of 503) and $29 \%$ (144 of 503) of patient procedures, respectively. Intraoperative tissue cultures were performed for $32 \%$ (160 of 503) of patient procedures. For all recorded variables, more complete preoperative and intraoperative data were collected for the PJI group when there was a high clinical suspicion for infection. Second, we do not have antecedent antibiotic administration data before culture acquisition because a high predominance of infected patients were referred from outside institutions and records are incomplete. We sought to mitigate the risk of bias induced by unequal pretreatment by directly comparing procedures with all three culture types available. This subgroup analysis, although informative, is prone to selection bias, because only 97 of 503 patient procedures (19\%) were available for direct comparison. All patient procedures with all three culture types were part of the PJI cohort (97 of 178 [55\%]) and were most likely to have presented with a clinically important PJI. An explanation for the lack of 
consistency in acquiring all three culture types includes variation in clinical management among surgeons and also along the timeline of data collection because preliminary results began to dictate that all three culture types should be obtained for more rigorous scientific comparison. This highlights a further limitation in that on implementation and throughout the majority of the data collection period, we did not have set criteria for how to determine which positive implant sonicate cultures were the result of infection versus contamination, thus contributing to variability of clinical management of these patients. In addition, currently we only have 2-year followup data on 25 of $53(47 \%)$ patients with positive implant sonication cultures taken during presumed aseptic revision procedures. Ongoing followup for the remaining patients over the short term as well as the entire cohort over the medium and long term is critical as we seek to correlate statistically optimized CFU/plate thresholds with real-world clinical outcomes. For example, should patients with $<5 \mathrm{CFU} /-$ plate present with a PJI without reason to suspect a new infectious source (and with the same organism), this would call into question the reliability of our CFU/plate PJI threshold.

Our research questions sought to help provide clarity on the relative accuracy of implant sonication cultures versus traditional culture methodologies. Across all procedures as well as within our subgroup analysis, we found the sensitivity of implant sonicate culture was greater than tissue culture and synovial fluid culture, whereas there was no difference in specificity. This was consistent with the findings of prior studies on implant sonication $[1,2,15]$. The sensitivity and specificity values reported here for implant sonicate cultures with more than $5 \mathrm{CFU} /$ plate are consistent with a recent meta-analysis of implant sonicate culture use in total joint arthroplasty [19]. As the largest study on implant sonication to date, our report helps to substantiate these earlier findings [19]. Additionally, although no perfect gold standard for PJI exists, the MSIS consensus criteria for diagnosis of PJI is the best standard currently available. Our study was the first to utilize the MSIS consensus criteria for defining PJI versus aseptic revision, helping to address previous concerns over misclassification bias by decreasing the likelihood of misattributing cases to either group [19].

Prior studies have identified coagulase-negative Staphylococcus, MRSA, and MSSA as the three most common organisms involved in PJIs [17]. Our study corroborates these earlier findings as well as those of other studies that identify that up to $50 \%$ of PJIs are associated with other less common bacterial species [4]. Routine use of implant sonicate cultures in addition to standard culture types identified a broad spectrum of bacterial species in PJIs and a surprisingly high proportion of aseptic revisions with bacteria present. The bacterial spectrum differed between the cohort of PJIs and those in which there was unexpected growth after surgery for aseptic indications. The trend within the aseptic revision cohort was for a predominance of low-virulence organisms not typically associated with a robust host inflammatory response.

In addition, we sought to clinically follow those patients who had positive implant sonicate cultures but did not meet the threshold of a MSIS consensus criteria infection with the intent of providing a real-world correlate of statistically determined optimizing CFU/plate threshold values. Bacteria were identified by implant sonicate culture in $16 \%$ of presumed aseptic revision procedures with roughly onethird receiving antibiotic treatment for infection (Table 6). Regarding the clinical followup of presumed aseptic negative procedures with positive implant sonicate cultures, our mean clinical followup was 22 months for the entire cohort. At this time, 25 of $53(47 \%)$ have 2-year clinical followup available. Three of $15(20 \%)$ patients with less than $5 \mathrm{CFU} /$ plate received postrevision antibiotic therapy. In the cohort with more than $5 \mathrm{CFU} /$ plate on implant sonicate culture, four of $10(40 \%)$ received treatment for infection. Despite the small sample size and early returns of 2-year followup data in this subset of patients, the suggestion is that the clinical validity $>5 \mathrm{CFU} /$ plate threshold value remains unresolved despite statistical modeling across the entire subject population that would suggest it as an optimal threshold point.

Regular use of implant sonicate culture for presumed aseptic revision procedures may improve the accuracy of PJI diagnosis by detecting low-virulence organisms that otherwise would not grow in other culture types. Preoperative clinical evaluation for patients undergoing aseptic revision surgery in this study routinely incorporated most, if not all, components of the MSIS consensus criteria to exclude infectious causes. However, despite adhering to commonly accepted standards of preoperative evaluation, $>15 \%$ of aseptic revisions had positive implant sonicate cultures. One prior study found that synovial fluid culture is particularly valuable in detecting infection in procedures where there is unsuspected bacterial growth. Puig-Verdie et al. [11] in particular reported $100 \%$ sensitivity and $99 \%$ specificity in this clinical scenario, but they did not report the percentage of aseptic revisions with bacterial growth. The clinical importance of the presence of bacteria in this scenario is yet to be determined, but it prompts the question of whether more revision arthroplasty indications are actually the result of infectious etiologies. We have and will continue to follow the clinical course of those patients with positive cultures in the setting of aseptic revisions.

In conclusion, it is our recommendation that this simple, relatively low-burden test be utilized more broadly in conjunction with current PJI diagnostic standards as 
established by the MSIS. Unexpected positive cultures in patients who are undergoing revision arthroplasty may be indicative of inadequate screening procedures, culture methodologic issues (including false-positives), or an inability to detect low-virulence pathogens with traditional cultural techniques. However, the unexpectedly high rate of positive cultures in the absence of MSIS consensus criteria to diagnose PJI supports our clinical suspicion that a higher proportion of infections is likely responsible for, or at least a contributing factor to, so-called aseptic loosening than can be detected by other widely utilized means. Future directions of research might include a more formalized evaluation of the implant sonication-positive cohort with objective protocols for treatment versus observation. Additionally, we have already implemented a change in protocol to include all three culture types for both PJI and aseptic revisions to improve our ability to perform direct comparative analysis.

Acknowledgments We thank Dr William Pasculle (Director, University of Pittsburgh Medical Center clinical microbiology laboratories) and Dr Nalini Rao (Division of Infectious Diseases) for their role in establishing the implant sonication protocol at the University of Pittsburgh Medical Center. In addition, Dr Pasculle was a key contributor to the microbiologic methods section of our manuscript. Adam Popchak provided key support in the form of statistical analysis. Alma Heyl, Research Coordinator for the Division of Adult Reconstruction at the University of Pittsburgh Medical Center, was instrumental in data collection.

\section{References}

1. Atkins BL, Athanasou N, Deeks JJ, Crook DW, Simpson H, Peto TE, McLardy-Smith P, Berendt AR. Prospective evaluation of criteria for microbiological diagnosis of prosthetic-joint infection at revision arthroplasty. The OSIRIS Collaborative Study Group. J Clin Microbiol. 1998;36:2932-2939.

2. Bare J, MacDonald SJ, Bourne RB. Preoperative evaluations in revision total knee arthroplasty. Clin Orthop Relat Res. 2006;446:40-44.

3. Bergin PF, Doppelt JD, Hamilton WG, Mirick GE, Jones AE, Sritulanondha S, Helm JM, Tuan RS. Detection of periprosthetic infections with use of ribosomal RNA-based polymerase chain reaction. J Bone Joint Surg Am. 2010;92:654-663.

4. Cazanave C, Greenwood-Quaintance KE, Hanssen AD, Karau MJ, Schmidt SM, Gomez Urena EO, Mandrekar JN, Osmon DR, Lough LE, Pritt BS, Steckelberg JM, Patel R. Rapid molecular microbiologic diagnosis of prosthetic joint infection. $J$ Clin Microbiol. 2013;51:2280-2287.
5. Holinka J, Bauer L, Hirschl AM, Graninger W, Windhager R, Presterl E. Sonication cultures of explanted components as an add-on test to routinely conducted microbiological diagnostics improve pathogen detection. J Orthop Res. 2011;29:617-622.

6. Kempthorne JT, Ailabouni R, Raniga S, Hammer D, Hooper G. Occult infection in aseptic joint loosening and the diagnostic role of implant sonication. Biomed Res Int. 2015;2015:946215.

7. Nelson CL, McLaren AC, McLaren SG, Johnson JW, Smeltzer MS. Is aseptic loosening truly aseptic? Clin Orthop Relat Res. 2005;437:25-30.

8. Parvizi J, Gehrke $\mathrm{T}$, International Consensus Group on Periprosthetic Joint Infection. Definition of periprosthetic joint infection. J Arthroplasty. 2014;29:1331.

9. Parvizi J, Jacovides C, Zmistowski B, Jung KA. Definition of periprosthetic joint infection: is there a consensus? Clin Orthop Relat Res. 2011;469:3022-3030.

10. Portillo ME, Salvado M, Alier A, Martinez S, Sorli L, Horcajada JP, Puig L. Advantages of sonication fluid culture for the diagnosis of prosthetic joint infection. J Infect. 2014;69:35-41.

11. Puig-Verdie L, Alentorn-Geli E, Gonzalez-Cuevas A, Sorli L, Salvado M, Alier A, Pelfort X, Portillo ME, Horcajada JP. Implant sonication increases the diagnostic accuracy of infection in patients with delayed, but not early, orthopaedic implant failure. Bone Joint J. 2013;95:244-249.

12. Rak M, KavcIc M, Trebse R, Co RA. Detection of bacteria with molecular methods in prosthetic joint infection: sonication fluid better than periprosthetic tissue. Acta Orthop. 2016;87:339-345.

13. Ribera A, Morata L, Moranas J, Agullo JL, Martinez JC, Lopez Y, Garcia D, Cabo J, Garcia-Ramiro S, Soriano A, Murillo O. Clinical and microbiological findings in prosthetic joint replacement due to aseptic loosening. J Infect. 2014;69:235-243.

14. Shen H, Tang J, Wang Q, Jiang Y, Zhang X. Sonication of explanted prosthesis combined with incubation in BD bactec bottles for pathogen-based diagnosis of prosthetic joint infection. J Clin Microbiol. 2015;53:777-781.

15. Spangehl MJ, Masri BA, O'Connell JX, Duncan CP. Prospective analysis of preoperative and intraoperative investigations for the diagnosis of infection at the sites of two hundred and two revision total hip arthroplasties. J Bone Joint Surg Am. 1999;81:672-683.

16. Trampuz A, Piper KE, Jacobson MJ, Hanssen AD, Unni KK, Osmon DR, Mandrekar JN, Cockerill FR, Steckelberg JM, Greenleaf JF, Patel R. Sonication of removed hip and knee prostheses for diagnosis of infection. $N$ Engl $J$ Med. 2007;357:654-663.

17. Trampuz A, Zimmerli W. Prosthetic joint infections: update in diagnosis and treatment. Swiss Med Wkly. 2005;135:243-251.

18. Trebse R, Pisot V, Trampuz A. Treatment of infected retained implants. J Bone Joint Surg Br. 2005;87:249-256.

19. Zhai Z, Li H, Qin A, Liu G, Liu X, Wu C, Li H, Zhu Z, Qu X, Dai K. Meta-analysis of sonication fluid samples from prosthetic components for diagnosis of infection after total joint arthroplasty. J Clin Microbiol. 2014;52:1730-1736. 